\title{
Resectable Pancreatic Ductal Adenocarcinoma
}

National Cancer Institute

\section{Source}

National Cancer Institute. Resectable Pancreatic Ductal Adenocarcinoma. NCI

Thesaurus. Code C151995.

Pancreatic ductal adenocarcinoma amenable to surgical resection. 